\title{
Evaluation of two immunodiagnostic tests for early rapid diagnosis of leptospirosis in Sri Lanka: a preliminary study
}

\author{
Egwin J. Eugene ${ }^{1}$, Shiroma M. Handunnetti ${ }^{1}$, Shalini A. Wickramasinghe ${ }^{1}$, Thilini L. Kalugalage ${ }^{1}$, \\ Chathuraka Rodrigo ${ }^{2}$, Hasith Wickremesinghe ${ }^{3}$, Nandana Dikmadugoda ${ }^{3}$, Pranitha Somaratne ${ }^{4}$, \\ H Janaka De Silva ${ }^{5}$ and Senaka Rajapakse $2^{2^{*}}$
}

\begin{abstract}
Background: Leptospirosis is often treated based on clinical diagnosis. There is a need for rapid laboratory diagnosis for this condition. The aim of this study was to compare the diagnostic accuracy of two rapid IgM based immunodiagnostic assays with the microscopic agglutination test (MAT), in acute leptospirosis infection.

Methods: MAT, IgM based immunochromatographic test (Leptocheck-WB) and IgM ELISA were performed using acute sera of patients clinically suspected to have leptospirosis $(n=83)$. Bayesian latent class modeling was used to compare the accuracy of these tests.

Results: Percentage positivity for MAT, Leptocheck-WB, and IgM ELISA were 48.1, 55.3, and $45.7 \%$ respectively. Bayesian latent class modeling showed a combined positivity rate of leptospirosis of $44.7 \%$. The sensitivity of MAT, Leptocheck-WB and IgM ELISA were 91.4, 95 and $81.1 \%$, and specificity were 86.7, 76.4 and $83.1 \%$, respectively.

Conclusions: Leptocheck-WB has high sensitivity, and, because it is quick and easy to perform, would be a good screening test for acute leptospirosis infection. IgM ELISA has good specificity, and is comparable with MAT; given that it is easier to perform and more widely available than MAT, it would be a more appropriate confirmatory test for use in hospitals with limited access to a specialized laboratory.
\end{abstract}

\section{Background}

Leptospirosis is a zoonosis of ubiquitous distribution, caused by infection with pathogenic Leptospira species [1]. In Sri Lanka, disease notification data shows a steady increase in the incidence of leptospirosis over the last two decades, which is attributable to disease emergence as well as improved surveillance [2]. During a large outbreak which occurred in 2008, the reported incidence rate was 7099 cases (35.7 per 100,000 population), with 204 deaths. The disease continues to affect large numbers of people each year, especially those in the farming community, resulting in significant morbidity and economic impact. In 2013, there were 4308 reported cases,

\footnotetext{
* Correspondence: Senaka.ucfm@gmail.com

${ }^{2}$ Department of Clinical Medicine, Faculty of Medicine, University of

Colombo, Colombo 08, Sri Lanka

Full list of author information is available at the end of the article
}

with 78 deaths, giving an epidemiological prevalence of 21.5 per 100,000 population.

Clinical features of leptospirosis are similar to dengue and many other tropical infectious diseases in Sri Lanka; thus early laboratory confirmation of the diagnosis will help guide clinicians to institute appropriate treatment early in the course of the illness and plan appropriate resource allocation, potentially preventing complications and death. In most countries, accurate laboratory diagnosis is a challenge. Microscopic agglutination test (MAT) is generally considered the standard immunological test used for diagnosis of leptospirosis. Laboratory confirmation based on MAT is a much delayed process, and it is technically challenging, requiring experience laboratory scientists to perform the tests, and the maintenance of live leptospira cultures. Often, treatment is initiated on the basis of clinical assessment with subsequent laboratory confirmation using MAT. There 
is a need for rapid diagnostic tests which can yield accurate results early on in the course of the clinical illness. Clinical leptospirosis is a biphasic illness, with a leptospiraemic phase occurring from the $4^{\text {th }}$ to $7^{\text {th }}$ day followed by a leptospiruric phase that can last for 4-30 days. Leptospiruria coincides with the immune phase which begins with the appearance of $\operatorname{IgM}$ antibodies $[3,4]$. Therefore, IgM based serology is of diagnostic value during this phase. Many rapid serodiagnostic assays are available, such as IgM based microplate enzyme-linked immunosorbant assay (ELISA), IgM dot ELISA test, IgM dipstick, latex agglutination test and haemagglutination assay [5-8]. These rapid tests are easy to perform and read, although their scientific validity with respect to sensitivity and specificity need further evaluation. To date, only one study has been reported from Sri Lanka evaluating commercially available rapid immunodiagnostic kits [9]. This study evaluated the microplate ELISA (Institut Viron Serion GmbH, Germany) and showed very low sensitivity and specificity values. Other rapid serological diagnostic assays have not been evaluated against a reference test in Sri Lanka.

This study compared the efficacy of two rapid immunodiagnostic assays for the detection of leptospira IgM antibodies, i.e., IgM based immunochromatography test (Leptocheck-WB test) and IgM based microplate ELISA, together with MAT. MAT detects both IgM and IgG antibodies. The validity of MAT as a gold standard could be considered imperfect, as MAT positivity may result from previous infection, while a MAT-negative result in the presence of positivity by an IgM-specific assay may occur due to either low sensitivity or serovar specificity of MAT. In settings such as this, Bayesian latent class modeling has been suggested to be a more suitable method for evaluating diagnostic tests, as it assumes that all tests are imperfect $[7,10]$. We therefore compared these tests using Bayesian latent class modeling.

\section{Methods}

\section{Patients}

Serum samples were collected from patients clinically suspected to have acute leptospirosis, admitted to the $\mathrm{Na}$ tional Hospital, Colombo, (NHSL) and Base Hospital, Homagama (BHH), Sri Lanka during the period June to September 2010. The following criteria, based on World Health Organisation-Leptospirosis Epidemiology Research Group (WHO-LERG) Epidemiological criteria were used to define a suspected case of leptospirosis; acute febrile illness with any of the following: headache, myalgia, arthralgia, conjunctival suffusion, meningeal irritation, anuria, oliguria, proteinuria, jaundice, hemorrhages, cardiac arrhythmia, skin rash; or with a contact history of exposure to water or soil contaminated with urine of infected animals. Patients with a clear alternative diagnosis were excluded. MAT, IgM Leptocheck-WB test, and ELISA were performed on acute serum samples from these patients. Informed written consent was obtained from all patients included in the study. There were 83 patients presenting with clinically suspected acute leptospirosis; mean age 39.9 years ( $\mathrm{SD} \pm 15.2$ ), male: female ratio was 20:1.

\section{Laboratory methods}

The Leptocheck-WB test kit was obtained from Zephyr Biomedicals, India, and the IgM ELISA Leptospira kit from Diagnostic Automation Inc., USA. MAT was performed at the Department of Bacteriology, Medical Research Institute, Sri Lanka. For MAT, sera which gave an agglutination of at least $50 \%$ of the leptospires (compared with the control antigen) was considered to be positive, with a serum titre of $\geq 400$ considered the positive threshold [11]. Leptospira biflexa serovar Patoc strain Patoc-1, which is an indicator strain, was used as antigen in all three tests, i.e., MAT, Leptocheck-WB and IgM ELISA.

The Leptocheck-WB test kit components were used according to manufacturer's instructions. [13] Briefly, contents were placed at room temperature (RT; $25^{\circ} \mathrm{C}$ ), and the test device was labeled with the patient's identity (patient's code). Serum sample $(10 \mu \mathrm{l})$ was added into the sample port twice and 5 drops of sample running buffer was dispensed into the buffer port immediately. The device was kept at RT and the results were read at the end of $15 \mathrm{~min}$. Depending on various intensities observed, they were subjectively scored as $0,1+, 2+, 3+$ or $4+$ based on the intensity of colour of the antigen band, using a colour reference diagram. A result of $1+$ or greater was considered positive according to the manufacturers' instructions.

The antigen coated on the IgM ELISA kit used was whole cell lysate from Leptospira biflexa patoc-1. The IgM-ELISA kit was used according to manufacturer's instructions [10]. Briefly, 1:40 dilutions of serum samples were prepared using the dilution buffer provided with the ELISA kit. Rheumatoid factor (RF) absorbent $(40 \mu \mathrm{l})$ was added to $100 \mu \mathrm{l}$ of diluted test serum (patient sera and healthy control sera), mixed well, incubated in the tubes for $5 \mathrm{~min}$ and then added to ELISA plate. Prediluted negative and positive controls provided were added to the ELISA plate, and the RF absorbent added according to manufacturers' instructions. The plate was incubated at RT for $10 \mathrm{~min}$. The contents were then washed 3 times with the diluted wash buffer provided, and two drops of enzyme conjugate were added to each well and incubated at RT for $10 \mathrm{~min}$. The plate was washed 3 times with wash buffer and two drops of chromogen was added to each well and incubated at RT for $5 \mathrm{~min}$. The reaction was stopped by adding 2 drops of the stop solution per well, mixed well, and the plate 
was read at $450 \mathrm{~nm}$ using an ELISA plate reader (ELx800- universal microplate reader, Bio-Tek, Instruments INC, Canada.). The cut-off value was $\geq 0.848$, calculated by plotting the Receiver Operating Characteristic (ROC) curve.

\section{Statistical analysis}

Data was entered and analyzed using SPSS $^{\circ}$ statistical software version 17. Descriptive analysis was done using percentages. The MICE tool (Modelling for Infectious Diseases Centre, Mahidol-Oxford Research Unit, Thailand [http://mice.tropmedres.ac/home.aspx]) was used for Bayesian latent class modeling. The use of Bayesian latent class models to determine the accuracy of diagnostic tests where the gold standard is imperfect has been described elsewhere [7].

\section{Ethics statement}

Ethics approval (Ref No. EC/09/054) was obtained from the Ethics Review Committee of the Faculty of Medicine, University of Colombo.

\section{Results}

For the 83 patients included in the study, the positivity obtained by the three tests, i.e., MAT, immunochromatography and IgM ELISA were 48.1, 55.3, and $45.7 \%$ respectively. Positivity by all three tests was $32.5 \%$. The combined positivity for Leptocheck-WB and MAT was $41.0 \%$ whereas positivity with Leptocheck-WB and IgM ELISA was $37.3 \%$ and IgM ELISA and MAT was $34.9 \%$ (Table 1).

Based on Bayesian latent class modeling, the combined positivity rate (i.e., percentage positive based on any of the tests) of leptospirosis was $44.7 \%$ (95\% Credible Interval [CrI] 30.8-60.8). The sensitivity of MAT, Leptocheck-WB and IgM ELISA were 91.4, 95 and $81.1 \%$, and specificity were $86.7,76.4$ and $83.1 \%$, respectively. Leptocheck-WB had the highest sensitivity,

Table 1 Detection of anti-leptospira antibodies in human sera, by MAT, Leptocheck-WB and IgM ELISA assays

\begin{tabular}{cccc}
\hline Number of samples by test (\%) & MAT & Leptocheck-WB & IgM ELISA \\
\hline $27(32.5)$ & + & + & + \\
$7(8.4)$ & + & + & - \\
$2(2.4)$ & + & - & + \\
$4(4.8)$ & + & - & - \\
$4(4.8)$ & - & + & + \\
$8(9.6)$ & - & + & - \\
$6(7.2)$ & - & - & + \\
$26(31.3)$ & - & - & - \\
Total $83(100.0)$ & & & $46.9 \%$ \\
\hline Positivity by single test & $48.1 \%$ & $55.4 \%$ & \\
\hline
\end{tabular}

with a negative predictive value of $95.1 \%$. MAT still had the highest specificity, although the specificity of IgM ELISA was comparable (positive predictive values 84.7 and $79.6 \%$ ) (Table 2). Good convergence was also seen for all parameters by using the MICE tool (see methods). Bayesian p-values for the profiles ranged from 0.4180.630 , indicating good fitness.

\section{Discussion}

The primary objective of this preliminary study was to compare the relative accuracy and suitability of two rapid immunodiagnostic tests, i.e., an IgM based immuno-chromatographic test, i.e., Leptocheck-WB, and a microplate IgM ELISA, together with the conventional MAT test which is used most widely for diagnosis currently.

Leptocheck-WB is easy to perform, is a rapid method which takes only $15 \mathrm{~min}$, and requires only a single dilution with no requirement of special equipment. In contrast, IgM ELISA has several steps in its procedure with a time duration of about 50-60 min and requires an ELISA plate reader. Leptocheck-WB test gave consistent results and the bands were stable for more than 12 months. Both tests were relatively inexpensive with a cost of less than 200 SLR (1.6 USD/test). The positivity produced by Leptocheck-WB test was $55.3 \%$ while that for IgM ELISA was $45.7 \%$. We suggest, based on our findings, that Leptocheck-WB would be an appropriate

Table 2 Comparison of diagnostic accuracy of MAT, Leptocheck-WB, and IgM ELISA, using Bayesian latent class modeling

\begin{tabular}{lc}
\hline Parameters & $\begin{array}{l}\text { Bayesian latent } \\
\text { class model \% } \\
(95 \% \text { credible interval) }\end{array}$ \\
\hline Combined positivity rate & $44.7(30.8-60.8)$ \\
MAT & $91.4(72.2-100)$ \\
Sensitivity & $86.7(70.7-99.3)$ \\
Specificity & $84.7(63.6-99.3)$ \\
PPV & $92.7(71.2-100)$ \\
NPV & \\
Leptocheck-WB & $95.0(79.3-100)$ \\
Sensitivity & $76.4(60.8-93.2)$ \\
Specificity & $76.5(56.2-94.3)$ \\
PPV & $95.1(75.9-100)$ \\
NPV & \\
IgM ELISA & $81.1(62.4-97.3)$ \\
Sensitivity & $83.1(68.8-94.8)$ \\
Specificity & $79.6(60.4-94.4)$ \\
PPV & $84.5(64.0-98.2)$ \\
NPV &
\end{tabular}


screening test for leptospirosis, especially in hospitals with limited laboratory facilities. Leptocheck-WB can easily be performed as an individual test, either with serum or whole blood samples, the assay contents are stable and could be transported and stored at ambient temperatures. The test is a portable package, and is easy to interpret, with no requirement for skills in handling specialized equipment such as an ELISA microplate reader. Previous studies have shown variable sensitivity and specificity of Leptocheck-WB. One study in South Gujarat showed Leptocheck-WB to have relatively low sensitivity (78.7) and higher specificity (88.3\%) [12], compared to the sensitivity and specificity of 95 and $76.5 \%$ in our study. In contrast, another study, also in South Gujarat, showed Leptocheck-WB to have very high sensitivity (98.4\%), while specificity was similar to the previous study (86.9\%) [11].

Despite the high specificity and sensitivity shown with MAT, this test is more difficult to perform, requires a specialized laboratory and trained staff, and is thus not available routinely to clinicians in remote rural hospitals. Current WHO guidelines recommend a MAT titer of $\geq 400$ in a single or paired serum sample or a 4-fold increase in MAT in acute and convalescent sera for laboratory conformation of Leptospirosis [13]. In our study, a MAT titer of $\geq 400$ in acute serum was considered as the reference standard. However, MAT measures both IgG and IgM; the duration for which IgG and IgM levels persist after acute infection is not clearly known. Thus, MAT positivity could reflect previous rather than acute infection. Our own data suggests that IgM levels decrease rapidly 3 months after acute infection (unpublished data). We suggest that IgM ELISA would be a more suitable test than MAT for use by clinicians treating patients with acute infections, for the following reasons. Firstly, the fact that MAT is only available in larger laboratories leads to delays and difficulties in obtaining early results, while IgM ELISA is more likely to be available at the point of care. Secondly, there is mounting evidence that IgM ELISA maybe more sensitive in identifying infection early on in the course of illness, which will provide a definite advantage to the treating clinician to prioritize healthcare resources in a timely manner. A meta-analysis of studies of ELISA in leptospirosis showed that the pooled sensitivity for IgM ELISA tests was 80.4 (79.2-81.5), and specificity was 94.4 \% (93.9-94.9) [14], values which are similar to our study. Nonetheless, there is considerable heterogeneity seen in ELISA tests for leptospirosis, in previous studies [14]. The fact that the commercial ELISA method used in our study has good sensitivity and specificity is of importance in the local context.

Arguably, the main limitation of these three tests is the fact that they are genus specific for Leptospira biflexa. There is considerable interest in developing serovar specific immunological tests, and also in-house immunodiagnostics which would give higher diagnostic specificity and sensitivity for local serovars. Nonetheless, our results show reasonably high accuracy with these commercial tests, making these pragmatic alternatives to guide clinicians treating patients with leptospirosis in resource limited developing country settings.

Interestingly, pooled meta-analysis shows that, based on ELISA, IgM antibodies are more specific that IgG antibodies for leptospirosis [14]. It is postulated that this could be due other febrile illnesses causing a nonspecific rise in IgG, resulting in false positives. If this were the case, it would also suggest that MAT, which tests both IgG and IgM antibodies, is less specific than IgM. Nonetheless, MAT is likely to remain the epidemiological gold standard for diagnostic confirmation of leptospirosis infection, eventually to be replaced by genomic techniques.

This was a preliminary study with limited sample size, and our findings emphasize the need for a larger study with greater statistical power. Such a study is currently in progress by our research team. This study was limited to one season and emphasizes the need for coverage of different geographical regions of the country, a longer study period to include seasonal variations and a larger sample size. Factors that could affect the reported sensitivity and specificity of Leptocheck-WB test may include its genus specific nature and inability to react and recognize the infecting serovar specific IgM antibodies.

\section{Conclusions}

Our preliminary findings suggest that Leptocheck-WB would be a suitable screening test, and IgM ELISA an appropriate confirmatory test for patients with acute leptospirosis. MAT will remain the reference standard for epidemiological purposes, especially if PCR is not available, however it's availability is limited.

\section{Abbreviations \\ MAT: Microscopic agglutination test; IgM: Immunoglobulin M; \\ IgG: Immunoglobulin G; PCR: Polymerase chain reaction; ELISA: Enzyme- linked immunosorbant assay; RT: Room temperature; MICE: Modeling for infectious diseases centre; Crl: Credible interval.}

\section{Competing interests}

The authors declare that they have no competing interests.

\section{Authors' contributions}

SMH conceived the study. EJE, SAW, HJDeS, SR and SMH designed the study. $C R, H W, N D$ and SR did the clinical assessment. EJE carried out the IgM ELISA and Leptocheck-WB assays. EJE, SAW and SMH analyzed and interpreted these findings. PS and TLK carried out and interpreted the MAT data. EJE wrote the first draft; SR made critical revisions; all authors critically revised the manuscript for intellectual content, read and approved the final version. EJE, SMH and SR are guarantors of the paper. 


\section{Acknowledgements}

We thank the Director/Medical Superintendent, Consultants, Registrars, House Officers, Sisters \& Nurses of National Hospital Sri Lanka and Base Hospital Homagama, Sri Lanka, Staff of Medical Research Institute, Dr Rohini Gunaratne, Dr Thamal Vithanage, and all patients \& individuals who donated blood for this study. This work was supported by the Institute of Biochemistry, Molecular Biology and Biotechnology, University of Colombo, Sri Lanka.

\section{Author details}

'Institute of Biochemistry, Molecular Biology and Biotechnology, University of Colombo, Colombo 03, Sri Lanka. ${ }^{2}$ Department of Clinical Medicine, Faculty of Medicine, University of Colombo, Colombo 08, Sri Lanka. ${ }^{3}$ Base Hospital, Homagama, Colombo, Sri Lanka. ${ }^{4}$ Department of Bacteriology, Medical Research Institute, Colombo 08, Sri Lanka. ${ }^{5}$ Department of Medicine, Faculty of Medicine, University of Kelaniya, Ragama, Sri Lanka.

Received: 7 February 2015 Accepted: 4 August 2015

Published online: 11 August 2015

\section{References}

1. Agampodi SB. Case definitions in Leptospirosis: a note to Sri Lankan researchers. Sri Lankan J Infect Dis. 2012;2(2):55-7.

2. Agampodi SB, Peacock SJ, Thevanesam V, Nugegoda DB, Smythe L, Thaipadungpanit J, et al. Leptospirosis outbreak in Sri Lanka in 2008: lessons for assessing the global burden of disease. AmJTrop Med Hyg. 2011;85(3):471-8.

3. Bajani MD, Ashford DA, Bragg SL, Woods CW, Aye T, Spiegel RA, et al. Evaluation of four commercially available rapid serologic tests for diagnosis of leptospirosis. J Clin Microbiol. 2003;41(2):803-9.

4. Bal AM. Unusual clinical manifestations of leptospirosis. J Postgrad Med. 2005;51(3):179-83.

5. DebMandal M, Mandal S, Pal NK. Serologic evidence of human leptospirosis in and around Kolkata, India: a clinico-epidemiological study. Asian Pacific J Tropical Med. 2011;4(12):1001-6.

6. Effler PV, Bogard AK, Domen HY, Katz AR, Higa HY, Sasaki DM. Evaluation of eight rapid screening tests for acute leptospirosis in Hawaii. J Clin Microbiol. 2002;40(4):1464-9.

7. Limmathurotsakul D, Turner EL, Wuthiekanun V, Thaipadungpanit J, Suputtamongkol Y, Chierakul W, et al. Fool's gold: Why imperfect reference tests are undermining the evaluation of novel diagnostics: a reevaluation of 5 diagnostic tests for leptospirosis. Clin Infect Dis. 2012;55(3):322-31.

8. Gussenhoven GC, van der Hoorn MA, Goris MG, Terpstra WJ, Hartskeerl RA, Mol BW, et al. LEPTO dipstick, a dipstick assay for detection of Leptospiraspecific immunoglobulin M antibodies in human sera. J Clin Microbiol. 1997;35(1):92-7.

9. Agampodi SB, Thevanesam V, Senaratne T. Validity of a commercially available IgM ELISA test for diagnosing acute leptospirosis in high endemic districts of Sri Lanka. Sri Lanka J Infect Dis. 2014;4:83-9.

10. Limmathurotsakul $D$, Jamsen $K$, Arayawichanont $A$, Simpson JA, White $L$, Lee $\mathrm{SJ}$, et al. Defining the true sensitivity of culture for the diagnosis of melioidosis using Bayesian latent class models. PLoS One. 2010;5, e12485.

11. Panwala T, Mulla S, Patel P. Seroprevalence of leptospirosis in south gujarat region by evaluating the two rapid commercial diagnostic kits against the mat test for detection of antibodies to leptospira interrogans. National J Comm Med. 2011;2:64-70.

12. Sehgal SC, Vijayachari P, Sharma S, Sugunan AP. LEPTO Dipstick: a rapid and simple method for serodiagnosis of acute leptospirosis. Trans R Soc Trop Med Hyg. 1999;93(2):161-4.

13. WHO. Report of the second meeting of the leptospirosis burden epidemiology reference group. Geneva, Switzerland: WHO; 2011

14. Signorini ML, Lottersberger J, Tarabla HD, Vanasco NB. Enzyme-linked immunosorbent assay to diagnose human leptospirosis: a meta-analysis of the published literature. Epidemiol Infect. 2013;141(1):22-32.

\section{Submit your next manuscript to BioMed Central and take full advantage of:}

- Convenient online submission

- Thorough peer review

- No space constraints or color figure charges

- Immediate publication on acceptance

- Inclusion in PubMed, CAS, Scopus and Google Scholar

- Research which is freely available for redistribution 